9-1999

\title{
Variación Fonológica y Estilística en el Español de Puerto Rico
}

Antonio Medina-Rivera

Cleveland State University, a.medinarivera@csuohio.edu

Follow this and additional works at: https://engagedscholarship.csuohio.edu/clmlang_facpub

Part of the Spanish Linguistics Commons

How does access to this work benefit you? Let us know!

Publisher's Statement

Published by: American Association of Teachers of Spanish and Portuguese

\section{Recommended Citation}

Medina-Rivera, Antonio. "Variación Fonológica Y Estilística En El Español De Puerto Rico." Hispania. 82.3 (1999): 529-541.

This Article is brought to you for free and open access by the Department of World Languages, Literatures, and Cultures at EngagedScholarship@CSU. It has been accepted for inclusion in World Languages, Literatures, and Cultures Faculty Publications by an authorized administrator of EngagedScholarship@CSU. For more information, please contact library.es@csuohio.edu. 


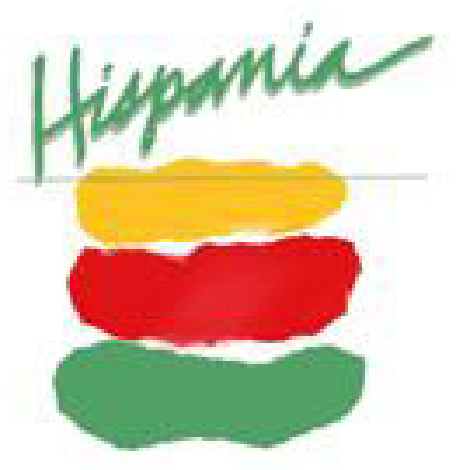

Variación fonológica y estilística en el español de Puerto Rico Author(s): Antonio Medina-Rivera

Source: Hispania, Vol. 82, No. 3 (Sep., 1999), pp. 529-541

Published by: American Association of Teachers of Spanish and Portuguese

Stable URL: http://www.jstor.org/stable/346322

Accessed: 29/05/2013 10:06

Your use of the JSTOR archive indicates your acceptance of the Terms \& Conditions of Use, available at http://www.jstor.org/page/info/about/policies/terms.jsp

JSTOR is a not-for-profit service that helps scholars, researchers, and students discover, use, and build upon a wide range of content in a trusted digital archive. We use information technology and tools to increase productivity and facilitate new forms of scholarship. For more information about JSTOR, please contact support@jstor.org. 


\title{
Variación fonológica y estilística en el español de Puerto Rico
}

\author{
Antonio Medina-Rivera \\ Cleveland State University
}

\begin{abstract}
Se examina la correlación que existe entre dos variables fonológicas y tres variables estilísticas en el español de Puerto Rico. Las variables estilísticas que se tomaron en consideración son la situación (entrevista individual, conversación en grupo y una presentación oral), el discurso y la relación que existe entre hablante e interlocutor. Se concentra en el habla de 20 jóvenes adultos que residen en Caguas, Puerto Rico. Las variables fonológicas que se seleccionaron para este estudio son la vibrante múltiple (rr) y la vibrante simple (r) en posición de final de sílaba. Los resultados se sometieron al programa estadístico VARBRUL y las conclusiones muestran la importancia que tienen las variables estilísticas en la investigación sociolingüística.
\end{abstract}

Key Words: variación lingüística, variación estilística, variación fonológica, español de Puerto Rico

\section{Introducción}

En el presente estudio se examina la correlación que existe entre dos variables fonológicas del español de Puerto Rico y cuatro variables estilísticas. El estudio de López-Morales sobre variables fonológicas en el español de San Juan de Puerto Rico (1983) sirve como punto de partida para esta investigación. Sin embargo, en vez de limitarme a las variables labovianas tradicionales, tal como lo hace López-Morales, he optado por examinar otras variables estilísticas que aún no se han explorado en los estudios lingüísticos.

En general, los estudios sociolingüísticos sobre el español de Puerto Rico carecen de un análisis estilístico sustancioso y extensivo. Algunas excepciones iluminadas por los estudios de Labov son Poplack (1980), la cual contrasta el estilo informal en oposición al formal en el habla de sus informantes, y López-Morales (1983), el cual incorpora otros dos estilos: la lectura de una lista de palabras y la lectura de un pasaje. Sugiero a partir de la la siguiente investigación que los estudios sociolinguísticos que incorporen variables estilísticas incluyan nuevas variables que resulten más apropiadas para examinar las diferencias que existen en el habla. Debe tomarse en consideración el papel que juegan los hablantes en una conversación al igual que las diferentes situaciones en las que participa un hablante durante su conversación cotidiana.

Para la presente investigación se seleccionaron la vibrante múltiple (rr) y la (r) en posición final de sílaba, ya que el español de Puerto Rico es muy conocido tanto por la variante velar de (rr) como por la variante lateral de (r) en posición final de sílaba. Esta distinción es constantemente estereotipada por otros hablantes de español de otras partes de Latinoamérica y los Estados Unidos que han tenido algún contacto con el español de Puerto Rico. Ambas variantes fonológicas se consideran dentro del habla no estándar no sólo en Latinoamérica sino también en el mismo Puerto Rico. Desde una perspectiva social estas variantes han sido asociadas al habla rural de ciertas áreas de Puerto Rico y por lo tanto resultan muy apropiadas para el objetivo principal de esta investigación, que es examinar el habla de varios hablantes a partir de variables estilísticas.

La variación estilística se podría definir como las diferencias fonológicas, morfológicas, sintácticas y léxicas que existen cuando un mismo hablante se expone a diferentes situaciones linguísticas, en otras palabras a lo que llama Bell (1984: 145) variación intrapersonal. La variación estilística implica, por lo tanto, que ningún hablante 
se expresa de la misma manera en todas las situaciones a las que se enfrenta y que el hablante, hasta cierta medida, escoge las formas lingüísticas que quiere utilizar en determinada situación.

En la siguiente investigación se examinan dos de las variables fonológicas más estudiadas en la variedad del español puertorriqueño, pero esta vez desde una perspectiva de la variación estilística inspirada en el modelo sociolinguiístico de Bell. Este modelo de estilo de acuerdo a la audiencia (1984), el cual no es más que un modelo de acomodación lingüística en la que se incoporan variables para el análisis estilístico, ofrece una nueva dimensión para los estudios sociolingüísticos. Las variables estilísticas que serán tratadas no se han explorado exhaustivamente ni siquiera para los estudios del idioma inglés a los que generalmente se refiere Bell en sus investigaciones. Este modelo parece ser una explicación simple y adecuada para analizar las diferencias estilísticas que existen en un hablante y sugiere que los hablantes generalmente responden o reaccionan de acuerdo a las personas que les rodean, diseñando su estilo de acuerdo a esa audiencia con la que cuentan. Las variables estilísticas que se toman en consideración en el modelo de diseño de acuerdo a la audiencia no descartan la interacción y los roles que juegan los hablantes en el transcurso de una conversación; en este sentido son variables linguiísticas que dependen de la interacción de los hablantes.

En su estudio, Bell añade además otras variables que sirven de complemento a su modelo de estilo de acuerdo a la audiencia, a las que denomina como factores independientes de la audiencia o de la interacción de los hablantes. Estos factores se refieren a variables no personales como es el caso del lugar en donde se desarrolla una conversación o el tema de conversación. En los estudios en los que se considera el lugar de la conversación, los hablantes se exponen a distintos ambientes o lugares como podrían ser el lugar del trabajo, la casa, la iglesia, etc., mientras que en los estudios en los que se toma en consideración el tema de conversación, el habla de los participantes se examina a partir de los distintos temas que se presentan en un diálogo. Aun cuando el modelo de Bell ofrece una nueva dimensión en el mundo de la investigación sociolinguística hay que recalcar que muchas de las variables que Bell sugiere todavía no se han examinado del todo.

\section{Metodología}

Las variables fonológicas que se seleccionaron para este estudio son la vibrante múltiple (rr) y la (r) en posición final de sílaba. Para la vibrante múltiple se examinaron dos variantes, una variante alveolar $[r]$, la cual se considera como la variante estándar; y la variante velar [B], la cual es una pronunciación altamente estigmatizada. Con respecto a la variable (r) en posición final de sílaba, se tomaron en cuenta las pronunciaciones que se consideran estándar en el español culto como es el caso de la variante alveolar [r] y la variante fricativa $[x]$; frente a las variantes no estándar como es el caso de la variante lateralizada [1], aspiración [h] y elisión [0]. Los estudios linguíisticos que analizan estas variantes generalmente se limitan a examinar actitudes lingüísticas o a ofrecer descripciones impresionistas con respecto a las variantes en cuestión, sin examinar exhaustivamente aquellos factores linguísticos o extralingüísticos que intervienen en la producción de una variante $u$ otra. En el presente trabajo se ofrecen algunas de las explicaciones linguiísticas, estilísticas y sociales que se deben tomar en consideración para analizar estas variables sociolingüísticas.

La metodología de este estudio se diseñó de manera tal que sólo se tomaran en consideración algunos estilos de habla que podrían aparecer naturalmente en el lenguaje oral, sin tener que recurrir a géneros escritos ni a ejercicios diseñados para producir un estilo más preciso y formal, como, por ejemplo el pedirle a un hablante que leyera una lista de palabras. Para analizar el estilo se ha tomado en consideración un 
factor dependiente de la audiencia, en este caso la relación entre el hablante y su interlocutor, y tres factores independientes de la audiencia: situación (conversación individual, conversación en grupo y presentación oral), tema de conversación y tipo de género discursivo.

Para las conversaciones individuales se seleccionaron 20 jóvenes adultos entre la edad de 21 a 35 años, con al menos un año de educación universitaria. Todos los participantes nacieron y se criaron en la zona urbana de Caguas, Puerto Rico. De los 20 participantes escogí a 10 hablantes conocidos por mí y a 10 hablantes no conocidos por mí con anterioridad. Con el término "hablante conocido" me refiero a hablantes con los cuales ya tenía una relación afectiva, como es el caso de amigos cercanos y familiares. Con esta variable se pretende observar la manera en la que un hablante responde linguíisticamente de acuerdo a la relación que tiene con su interlocutor. La mayoría de los estudios en los que se toma en consideración la variación estilística no muestra la relación que existe entre el hablante y su interlocutor, o entrevistador y entrevistado, y en muchos casos, éstos ni siquiera hablan el mismo dialecto. Con el presente estudio se muestra que la relación entre los hablantes podría contribuir a que un hablante seleccione una variante fonológica $\mathrm{u}$ otra. De entre los 10 hablantes conocidos se escogieron cuatro para participar en conversaciones en grupo, y de estos cuatro se escogieron dos para una presentación oral. Los dos hablantes escogidos para la presentación oral fueron hablantes que hacían este tipo de presentación como parte de la rutina de sus respectivos trabajos. Se procuró que la presentación oral no fuera una ponencia leída. Los resultados de este estudio se sometieron al análisis estadístico de
VARBRUL 2s.

\section{Resultados}

Para el análisis de (r) en posición final de sílaba se incluyó una muestra de 6,635 casos y para el análisis de la vibrante múltiple (rr) se tomó en consideración una muestra de 1,894 casos. Con respecto a la variable (r) en posición final de sílaba se organizaron las variantes en dos grupos: variantes estándar (variante alveolar y variante fricativa), en oposición a las variantes no estándar (variante lateralizada, aspiración y elisión). Para el estudio de la vibrante múltiple ( $\mathrm{rr}$ ) se incluyeron las dos variantes antes mencionadas (variante alveolar vs. variante velar) a las que también se les denominó como variante estándar y variante no estándar respectivamente. La tabla 1 muestra las frecuencias para cada una de las variantes.

En ambos estudios, las variantes estándar ocurrieron con mayor frecuencia, $63.9 \%$ y $90.7 \%$ respectivamente, lo cual implica que estas variantes siguen siendo la norma en el español de Puerto Rico. Cabe mencionar además, con respecto a las variantes no estándar de la variable (r) en posición de final de sílaba, que del $36.1 \%$ de los casos señalados en la tabla 1, el $92.4 \%$ corresponde a la variante lateralizada, con lo cual se muestra que la aspiración y la elisión no son tan frecuentes para el español de Puerto Rico, como también lo muestra López-Morales (1983). En el estudio de la variante múltiple ( $\mathrm{rr}$ ) se muestra que la velarización no alcanza los mismos niveles de ocurrencia que la lateralización. Además, cabe señalar que mientras todos los hablantes produjeron la variante lateralizada, hubo hablantes que no produjeron del todo la variante velar, los cuales, por tener

Tabla 1

FRECUENCIA DE LAS VARIANTES

\begin{tabular}{|c|c|c|c|c|}
\cline { 2 - 5 } \multicolumn{2}{c|}{} & \multicolumn{2}{c|}{ VARIABLE (r) } & \multicolumn{2}{c|}{ VARIABLE (rr) } \\
\hline Variantes estándar & {$[r],[d]$} & $63.9 \%$ & {$[r]$} & $90.7 \%$ \\
\hline Variantes no estándar & {$[l],[h],[0]$} & $36.1 \%$ & {$[\mathrm{~b}]$} & $9.3 \%$ \\
\hline Para la Variable $(r)$ & $\begin{array}{l}\mathrm{N}=6635 \\
\mathrm{~N}=1894\end{array}$ \\
Para la Variable $(\mathrm{r} r)$ & $\mathrm{n})$
\end{tabular}


la variante alveolar, se eliminaron en el análisis estadístico. Otra observación interesante es que los hablantes adultos (de 36 años o más) incluidos en Medina-Rivera $(1996,1997)$ mostraron frecuencias mucho más altas para la variante velar que los jóvenes adultos del presente estudio. Sin embargo, esta observación no es suficiente para sugerir que el proceso de velarización esté disminuyendo, dado el caso que hay hijos que producen la variante velar más que o tan frecuentemente como sus padres.

\subsection{Factores sociales y lingüísticos}

Para el análsis de la variable (r) en posición final de sílaba, tres factores lingüísticos produjeron resultados altamente significativos $^{1}$ de acuerdo al programa VARBRUL: carácter átono o tónico de la sílaba, el valor morfemático de (r) y el número de sílabas en la palabra. Las sílabas tónicas, en oposición a las átonas, son más propensas para la producción de variables no estándar, con una probabilidad de .57; las palabras que no son verbos infinitivos y la palabra porque produjeron probabilidades para las variantes no estándar de .56 y .53, respectivamente. El entorno fonológico no resultó ser un factor significativo para este estudio. Como única obsevación relacionada al entorno fonológico sería válido mencionar, sin embargo, que el proceso de lateralización no ocurre en posición intervocálica (e.g., caro), pero sí ocurre en posición final de palabra seguida de una palabra que empieza con vocal (e.g., por amor), lo cual se suele considerar como equivalente al entorno intervocálico. Este comentario es significativo en el sentido de que cuando se estereotipa el habla de un puertorriqueño en el exterior se exagera el proceso de lateralización llevándolo a entornos donde nunca ocurre (e.g. pelo en lugar de pero).

Los tres grupos de factores que resultaron significativos en el estudio de la variable (r) en posición final de sílaba demuestran que la palabra porque, las sílabas tónicas y las palabras de una o dos sílabas favorecen la producción de las variantes no estándar. La palabra porque es una palabra que ocurre con mucha frecuencia, favore- ciendo el uso de las variantes no estándar. Otra palabra que parece seguir un patrón similar, aunque no se sometió al análisis estadístico, es la palabra verdad. Palabras como porque y verdad ocurren con mucha frecuencia en la muestra; además son palabras que suelen producirse con bastante rapidez. Tanto verdad como porque se incluyeron entre las palabras de dos sílabas,las cuales también favorecen la producción de variantes no estándar. Las sílabas tónicas también favorecieron la producción de las variantes no estándar para este estudio (e.g. carta $>$ [kál-ta]). Las sílabas tónicas resultan o se perciben como más prominentes en el habla, lo cual hace del proceso de lateralización un fenómeno bastante prominente también. Esto conduce a que los hablantes de otras variedades de español que no tienen en su repertorio fonético la variante lateralizada oigan con facilidad la lateralización en boca de un puertorriqueño y lo crean incapaz de producir la variante alveolar.

Para el análisis de la vibrante múltiple, el único factor lingüístico ${ }^{2}$ que resultó significativo fue el tipo de palabra. "Otras palabras" en oposición a "nombres propios" favoreció la variante no estándar con una probabilidad de .52. Los nombres propios favorecieron la producción de la variante alveolar si se comparan a "otros nombres" o a "otras palabras." Esto podría deberse a que los nombres propios generalmente suelen ser el centro de foco en una conversación y por lo tanto el hablante suele ser mucho más cuidadoso al pronun-ciarlos. (Las probabilidades estadísticas de todos los factores se detallan en la tabla 5.)

En el presente estudio la interpretación de las variables sociales, ${ }^{4}$ las cuales no produjeron resultados estadísticamente significativos, sirve al menos para iluminar el problema de los estereotipos y las generalizaciones que se hacen sobre el español puertorriqueño, tanto a nivel local como en el exterior. Las mujeres favorecieron el uso de las variantes estándar en el estudio sobre la variable (r) en posición final de sílaba, pero también favorecieron el uso de la variante velar en el estudio de la vibrante 
múltiple. Inclusive dos de las mujeres adultas (Medina-Rivera 1996, 1997) obtuvieron frecuencias muy altas para la variante velar. Los estudios sociolingüísticos generalmente muestran que las mujeres tienden a favorecer con mayor frecuencia el uso de variables estándar. Esta generalización es hasta cierto punto correcta para la siguiente investigación, aunque no se cuenta con números que señalen valores estadísticamente significativos.

El nivel educativo, tanto de los hablantes como de sus padres, tampoco arrojó valores significativos. Sin embargo, el mismo sirve para argumentar con respecto al estereotipo que sugiere que solamente las personas con poca educación son los que producen las variantes no estándar. Aun cuando los estereotipos y las generalizaciones con respecto al uso de las variantes no estándar, por parte de las personas que viven en áreas rurales o que carecen de un nivel educativo alto, permanecen presentes entre la población del país, cabe mencionar a raíz del presente estudio, que muchas personas de zonas urbanas y con un nivel educativo superior no son conscientes de la frecuencia con la que producen variantes no estándar en su habla contidiana. La mayoría de los hablantes cree que no produce estas variantes no estándar; sin embargo, a la hora de ser entrevistados y grabados, y al analizar sus respectivas grabaciones, los resultados muestran lo contrario.

Las presiones o exigencias que se dan en el ambiente del trabajo y los medios de comunicación influyen hasta cierta medida en la manera en la que las personas hablan. Este tipo de observación complica la percepción que se tiene sobre ciertos fenómenos linguísticos. Algunos de los programas favoritos de la televisión puertorriqueña tienden, por ejemplo, a exagerar el uso de ciertas variantes linguiísticas que cuentan con valor estigmatizado en la sociedad. Por otro lado, el español que se utiliza en las noticias de la televisión y entre muchos de los cantantes se caracteriza por tener una pronunciación estándar debido al entrenamiento que se les da a estos profesionales para mejorar su dicción. Algunas de la figuras políticas del país producen con cierta regularidad variantes no estándar, quizás para identificarse más con el "pueblo" y el habla popular de algunos sectores rurales y/o marginales. Estas observaciones corresponden al concepto de "estilo de acuerdo a la audiencia” propuesto por Bell (1984) y discutido en las secciones anteriores.

En el sistema escolar se tratan de corregir algunas de las formas no estándar que se dan en el español de Puerto Rico; sin embargo, muchos de los maestros que corrigen a sus estudiantes en términos teóricos, a la hora de hablar no se pueden escapar de usarlas. Estos elementos sociales que se han mencionado en esta sección corresponden también al concepto de "arbitraje" que menciona Bell (1984) en su estudio. Los árbitros del habla vienen de distintas direcciones; son todas aquellas influencias que permanecen en nuestro (in) consciente (e.g., maestros, padres) y que en términos prácticos son imposibles de medir para el investigador, pero que no se deben descartar en el análisis cualitativo.

Volviendo nuevamente al fenómeno de la velarización, no es mi intención eliminar la posibilidad de que el mismo tenga un origen en las zonas rurales, ni mucho menos que el fenómeno de velarización se esté disminuyendo con los años. En realidad, en el estudio de Hammond (1987) se muestra que el nivel de velarización en la zona rural del suroeste de Puerto Rico alcanza el 72\%, mucho mayor que el $14.1 \%$ que presenta López-Morales (1983) en su estudio realizado en San Juan o el 9.3\% que muestra el presente estudio para la ciudad de Caguas. Por otro lado, si se comparan los resultados de Hammond, puede observarse que también es mayor que el $59 \%$ que presentó NavarroTomás en 1948, fecha en la cual la mayoría de la población de Puerto Rico era de origen rural.

\subsection{Variables estilísticas}

La contribución fundamental del presente estudio radica en la incorporación que se hace de nuevas variables estilísticas en la investigación sociolinguiística: la relación 
entre el hablante y su interlocutor, la situación, el discurso y el tema de conversación. Con estas variables, inspiradas en el modelo sociolingüístico de Bell (1984), se quiere dar un paso más allá de las distinciones tradicionales de Labov que contrastaban el habla formal vs. el habla informal, o el habla casual vs. el habla cuidadosa que caracterizan la mayoría de los estudios sociolingüísticos del español.

De las cuatro variables estilísticas que se incorporaron para la presente investigación, dos resultaron estadísticamente significativas tanto para el estudio de la variable (r) en posición final de sílaba como para el estudio de la vibrante múltiple. Estas variables o grupo de factores fueron el discurso y la situación. Aun cuando no todas las variables estilísticas resultaron estadísticamente significativas se procederá a discutir cada una de ellas, dada la importancia que las mismas podrían tener para futuras investigaciones que quieran explorar más a fondo la importancia de las variables estilísticas.

\subsubsection{La relación entre hablante e interlocutor}

Los hablantes conocidos favorecieron la producción de variantes no estándar con mayor frecuencia que los hablantes no conocidos. La tabla 2 muestra los resultados.

Estos resultados no reflejaron valores estadísticamente significativos de acuerdo al programa de VARBRUL. Sin embargo, resulta interesante observar las diferencias que existen entre los hablantes conocidos en oposición a los no conocidos; . 58 vs. .39 para las variantes no estándar de la variable (r) en posición de final de sílaba, y .51 vs. .49 para la variante no estándar de la vibrante multiple ( $\mathrm{rr}$ ) . La diferencia numérica que existe entre las probabilidades del estudio de la variable(r) en posición final de sílaba es mayor que la que existe entre los valores probabilísticos de la vibrante múltiple (rr), y cabe notar que en ambos estudios se reflejó que los hablantes conocidos tendieron a favorecer más el uso de variantes no estándar en comparación a los hablantes no conocidos.

Pienso que el rol del entrevistador, el cual se ha codificado para este estudio como conocido vs. no conocido, es fundamental para mostrar diferencias sociolinguiísticas. A esto se debe añadir además la personalidad del entrevistador, puesto que un entrevistador podría ser extremadamente serio y formal, o muy amigable y confiable. La personalidad del entrevistador podría afectar definitivamente el comportamiento lingüístico de un hablante no conocido. Otro elemento a considerar sería la edad del hablante y su interlocutor. En mi caso, seleccioné a hablantes de edad similar a la mía, lo que hasta cierto punto pudo haber minimizado el nivel de formalidad durante las entrevistas.

Los resultados de la tabla 2 corroboran la hipótesis de Bell con respecto a que los hablantes diseñan su habla de acuerdo a su

Tabla 2

RELACION ENTRE HABLANTE E INTERLOCUTOR

\begin{tabular}{|c|c|c|c|c|}
\hline & & & & \\
\hline & VARIA & (r) & \multicolumn{2}{|c|}{ VARIABLE (rr) } \\
\hline & \multicolumn{4}{|c|}{ Variantes Estándar } \\
\hline Conocido & \multicolumn{2}{|c|}{$55.6 \%$} & \multicolumn{2}{|c|}{$89.3 \%$} \\
\hline \multirow[t]{2}{*}{ No conocido } & \multicolumn{2}{|c|}{$75.6 \%$} & \multicolumn{2}{|c|}{$92.2 \%$} \\
\hline & \multicolumn{4}{|c|}{$\begin{array}{l}\text { Variantes No Estándar } \\
\text { VARBRUL }\end{array}$} \\
\hline Conocido & $44.4 \%$ & .58 & $10.7 \%$ & .51 \\
\hline No conocido & $24.4 \%$ & .39 & $7.8 \%$ & .49 \\
\hline
\end{tabular}


audiencia. En el caso de la presente investigación, la audiencia es [+/- familiar] para el entrevistador. Estos resultados sirven para mostrar las diferencias que existen dentro del repertorio lingüístico de un mismo hablante, tal como lo mostraron Rickford y McNair-Knox en su estudio de 1994. Ellos analizaron el habla de una joven afroamericana llamada Foxy. Esta produjo con mayor frecuencia formas vernáculas o no estándar cuando se entrevistó con otra joven amiga de su misma edad y raza, en comparación a un número menor de formas no estándar cuando se entrevistó con otra mujer desconocida, de raza blanca y la cual hablaba otro dialecto diferente al de Foxy. Cabe mencionar que en otra entrevista posterior entre Foxy y su amiga, Foxy produjo un número mucho menor de formas no estándar, aparentemente porque en el momento en el que ocurrió dicha entrevista Foxy estaba pasando por un momento de transición en su vida como adolescente. En esa ocasión Foxy había pasado por un cambio de vecindario y se encontraba estudiando en una escuela donde la mayoría de los estudiantes eran blancos, diferente a su escuela antigua en donde la mayoría de los estudiantes eran negros.

La relación entre el hablante y su interlocutor tiene un efecto en la variación linguística de los hablantes. Los factores "conocido" vs. "no conocido," diferencia entre el dialecto de los interlocutores y su raza, son variables que se deben tomar en consideración en los estudios sociolinguiísticos para examinar diferencias en el uso del lenguaje. Además, habría que tomar en consideración otros factores extralingüísticos que pertenecen a la historia del hablante, como es el caso de las personas que han influido en el repertorio linguiístico de dicho hablante, a las que Bell denomina "árbitros"(1984: 188-89).

En el español de Puerto Rico, y quizás en cualquier otro dialecto, la gente educada tiene la capacidad de producir formas estándar y no estándar en su habla, tal como se demuestra con los jóvenes adultos que participaron en el presente estudio. Es interesante notar que muchas de estas perso- nas negaron hacer uso de estas formas no estándar cuando se les consultó en un cuestionario de actitudes linguísticas (MedinaRivera 1997). Entre la gente educada las formas no estándar parecen reservarse para situaciones y audiencias muy específicas como es el caso de una conversación con amigos íntimos o familiares. La gente con un estatus socioeconómico menor generalmente tiende a tener frecuencias mayores de formas no estándar en su repertorio lingüístico, al menos eso es lo que muestran muchos de los estudios sociolingüísticos sobre variación. Mientras que la mayoría de los estudios sociolinguiísticos muestran que las clases educadas o de un nivel socioeconómico más alto favorecen con más frecuencia el uso de formas estándar, rara vez se señala de una manera explícita la posibilidad de que estos mismos hablantes puedan producir formas no estándar en determinadas situaciones. En general podría decirse que todo hablante está expuesto al uso de formas no estándar en el lenguaje. Por lo tanto sería un tanto inaceptable el ignorar la posibilidad de que todos los hablantes tengan la capacidad de producir formas no estándar en alguna de las situaciones lingüísticas a las que se enfrenta cada día. Se podría sugerir entonces, a partir de las observaciones anteriores, que cuando hablamos con familiares y amigos íntimos se disminuye la presión social para expresarnos con suma "corrección."

\subsubsection{Situación}

Además de observar la relación entre los interlocutores, es importante someter a varios de los hablantes a diferentes situaciones linguíisticas. Para el factor "situación" se escogió a cuatro de los hablantes "conocidos" (dos hombres y dos mujeres) para grabarlos en otras situaciones diferentes a la entrevista. A cada uno de los cuatro participantes se le grabó en situaciones diferentes de grupo. Para estas conversaciones de grupo se procuró que existiera un nivel de informalidad y que todos los participantes en la conversación en grupo se conocieran muy bien. Finalmente, a dos de los cuatro participantes (un hombre y una mujer) se 
les grabó mientras daban una presentación oral. Una de las presentaciones consistió en una charla educativa sobre higiene dental y la otra consistió en una presentación sobre el desarrollo y planificación de programas comunitarios. En ambas presentaciones, la audiencia fue un grupo de estudiantes universitarios. Ninguno de los dos presentadores eran profesores universitarios, pero parte de su rutina de trabajo incluía hacer este tipo de presentaciones. Yo no estuve presente para ninguna de las dos presentaciones, pero sí procuré que ambas cumplieran con los mismos requisitos: primero, que no fueran ponencias leídas y segundo, que la presentación fuera una tarea que el hablante hiciera como parte natural de su trabajo y que no fuera un situación artificiosa.

Para el factor situación se escogieron tres situaciones diferentes: la entrevista $o$ conversación individual, la conversación en grupo y la presentación oral. La situación de grupo fue espontánea; no se escogió ningún tema de antemano ni se escogieron preguntas para dirigir la conversación. En este sentido fueron diferentes a las conversaciones individuales o entrevistas, las cuales fueron semidirigidas con una lista de preguntas fijas para todos los participantes, aunque dejando siempre un espacio para la espontaneidad. Finalmente, para la presentación oral sí hubo una preparación y organización previa por parte del charlista. En términos de formalidad; las tres situaciones se podrían acomodar de la siguiente manera:

Formalidad de las Situaciones Grupo Individual Presentación Oral

El factor situación resultó altamente significativo de acuerdo al programa de VARBRUL. La tabla 3 muestra las frecuencias y probabilidades de ocurrencia.

Como era de esperar, el uso de variantes no estándar en la presentación oral resultó ser mucho menor en ambos casos, con probabilidades de ocurrencia de .16 y .40 , res- pectivamente. La probabilidad de ocurrencia de variantes no estándar aumentó drásticamente en el caso de la situación de grupo, .66 para la variable (r) en posición de final de sílaba y .85 para la vibrante múltiple.

Es interesante observar las diferencias que se dan para cada una de las situaciones que se incluyeron para la presente investigación. De acuerdo a Finegan y Biber (1994), las diferencias que existen entre una entrevista y un discurso público (equivalentes a la conversación individual y la presentación oral en el presente estudio) son mínimas. Ambas tienen como propósito informar sobre algún asunto y se producen al instante y con un cierto nivel de interacción con la audiencia, lo cual implica el uso de ciertos gestos y ademanes. Por otro lado, la entrevista y la conversación (equivalentes a la conversación individual y la conversación de grupo del presente estudio) también tienen sus parecidos, aunque difieren en cuanto a su propósito. La conversación en grupo es generalmente más afectiva y su punto de foco son las relaciones y no el proveer datos informativos. Mis resultados muestran sin embargo, que la conversación individual es más cercana a la conversación en grupo que a la presentación oral. El propósito de las tres situaciones coincide con lo que sugieren Finegan y Biber. No obstante las diferencias que existen entre sus comentarios y los míos podrían deberse a diferencias culturales entre la conversación de los hispanos y la conversación de los estadounidenses. Por otro lado, pienso que existe una diferencia fundamental entre una presentación oral/discurso público y una conversación individual/entrevista, la cual consiste en el tamaño y características de la audiencia. Cuando se compara una entrevista con una presentación oral, se puede notar que en una presentación oral hay mucha más gente, el charlista es el centro de atención y debe procurar ser muy cuidadoso en el manejo de su lenguaje para lograr que todos los asistentes lo entiendan con claridad (especialmente cuando no todas las personas de la audiencia manejan el mismo dialecto), y su expresión oral resul- 


\begin{tabular}{|c|c|c|c|c|}
\hline & \multicolumn{4}{|c|}{$\begin{array}{ll}\text { Tabla } 3 \\
\text { TIPO DE SITUACION } \\
\end{array}$} \\
\hline & \multicolumn{2}{|c|}{ VARIABIE (x) } & \multicolumn{2}{|c|}{ VARIABLE (rr) } \\
\hline \multicolumn{5}{|c|}{ Variantes Estándar } \\
\hline Grupo & \multicolumn{2}{|c|}{$37.2 \%$} & \multicolumn{2}{|c|}{$83.6 \%$} \\
\hline Individual & \multicolumn{2}{|c|}{$47.1 \%$} & \multicolumn{2}{|c|}{$91.4 \%$} \\
\hline Presentación & \multicolumn{2}{|c|}{$93.4 \%$} & \multicolumn{2}{|c|}{$94.7 \%$} \\
\hline \multicolumn{5}{|c|}{$\begin{array}{c}\text { Variantes No Estándar } \\
\text { VARBRUL }\end{array}$} \\
\hline Grupo & $62.8 \%$ & .66 & $16.4 \%$ & .85 \\
\hline Individual & $52.9 \%$ & .54 & $8.6 \%$ & .41 \\
\hline Presentación & $6.6 \%$ & .16 & $5.3 \%$ & .40 \\
\hline
\end{tabular}

ta ser mucho más enfática y con un tono de voz mucho más elevado. Tal como lo menciona Bell (1984), la audiencia parece tener un efecto mayor en el lenguaje y parece ser que los hablantes diseñan su habla de acuerdo a la audiencia que tienen.

Es necesario mencionar que la investigación de Finegan y Biber se concentra en el uso de formas lingüísticas [+/-elaboradas] o [+/-simplificadas] que se dan a lo largo de un continuo de registros orales y escritos. En el caso de la variación fonológica sugieren que los hablantes con un nivel socioeconómico más bajo tienden a usar variantes más simplificadas desde el punto de vista articulatorio. Ni la lateralización ni la velarización podrían considerárse como casos de simplificación articulatoria; más bien son variantes que implican un cambio en el modo o lugar de articulación. En este sentido no todas las formas linguiísticas no estándar son ejemplos de simplificación, tal como sugieren Finegan y Biber. También resulta un tanto contraproducente el comparar registros escritos con registros orales debido a las diferencias que existen en la preparación, corrección y motivaciones que se dan en la lengua escrita.

\subsubsection{Tipo de discurso}

Otra variable estilística a considerar en el presente estudio es el tipo de discurso, con lo cual se muestra que el habla no es homogénea en naturaleza, sino que cuando se analizan sus componentes, muestra una diversidad de complejidades en su estructura interna. Para esta variable se consideraron cinco géneros discursivos, a saber: diálogo, narrativa, descripción, exposición y argumentación. La tabla 4 muestra los resultados:

El diálogo y la narrativa favorecen más el uso de variantes no estándar cuando se les compara con otros tipos de géneros discursivos. Bajo la categoría "otros tipos de discurso" se agruparon los géneros descriptivo, expositivo y argumentativo, debido a que un primer análisis estadístico mostró probabilidades casi idénticas en cada género. Para el diálogo, la probabilidad de ocurrencia de las variantes no estándar fue de .59 en el caso de la variable (r) en posición final de sílaba y de .72 para la vibrante múltiple, mientras que para la narrativa las probabilidades de ocurrencia fueron de $.58 \mathrm{y}$ .56 , respectivamente.

Una de las razones para explicar el hecho de que el diálogo y la narrativa favorecen el uso de variantes no estándar mucho más que otros géneros discursivos podría deber- 
Tabla 4 TIPO DE DISCURSO

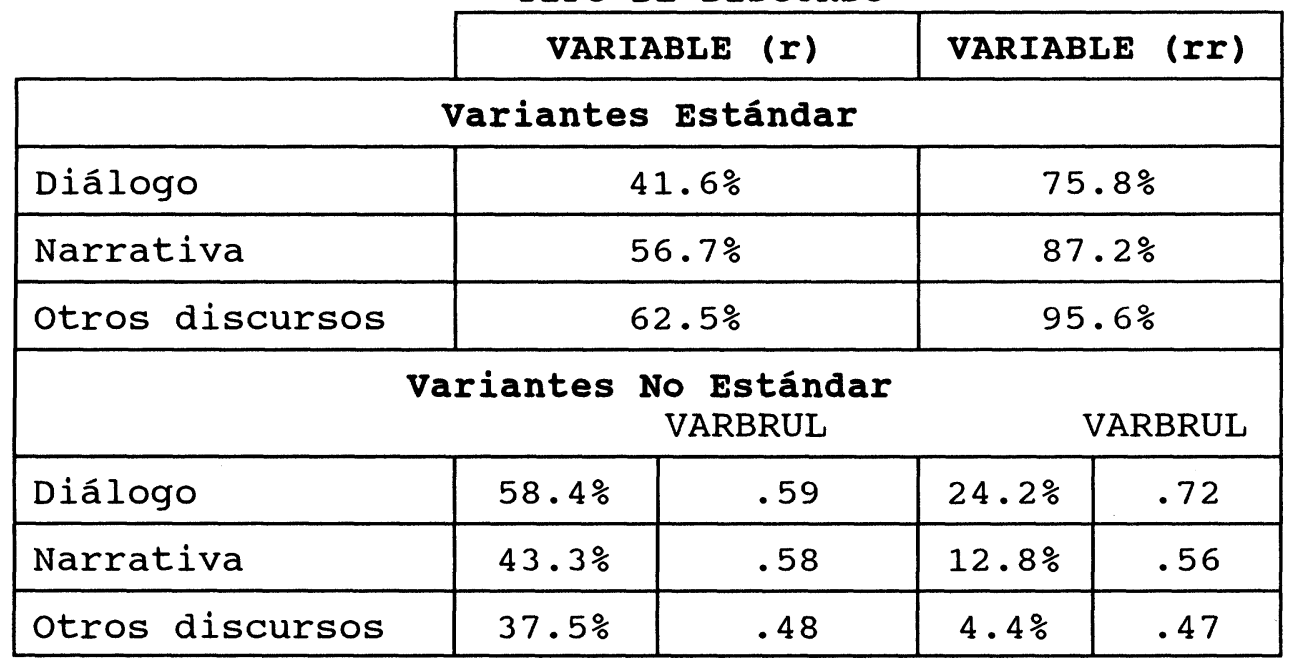

se al dinamismo y ritmo que caracterizan a ambos géneros discursivos. El diálogo, en este estudio, se define como una respuesta rápida y corta a una pregunta dentro de la conversación, sin la oportunidad de que el hablante desarrolle un patrón definitivo dentro de su discurso. La narrativa, por otro lado, se define como una secuencia de hechos que implican acción. La mayoría de las narrativas que se incluyeron en el presente estudio se refieren a experiencias personales con las que el hablante se identifica plenamente. En general podría decirse que no existe una narrativa sin la incorporación de emociones. Esta relación entre afectividad y la producción de variantes no estándar se mencionó anteriormente cuando se discutió la relación entre hablantes conocidos vs. hablantes no conocidos.

Finegan y Biber (1994), en su estudio sobre registros orales y escritos, muestran las diferencias que existen en términos de elaboración para cada uno de los registros que tomaron en consideración. Estas mismas diferencias pueden también observarse para cada uno de los estilos o géneros discursivos que se dan en la conversación. Finegan y Biber (1994: 322-25) observan diferencias con respecto al propósito del discurso, la oportunidad que pueda existir para la producción de un lenguaje cuidadoso y el grado o nivel de referente que comparten los hablantes o lectores. Estas mismas características podrían ser útiles para explicar las diferencias que existen para cada uno de los géneros discursivos que se dan en el lenguaje oral. Por ejemplo, tanto el género argumentativo como el descriptivo comparten una característica, que es la oportunidad que tiene el hablante para producir un habla mucho más cuidadosa y elaborada. La argumentación suele ocurrir con más lentitud en el habla; requiere un mayor nivel de procesamiento y organización, favoreciendo así con mayor frecuencia el uso de variantes estándar. La descripción, por otro lado, se nutre de una mayor ornamentación, sobre todo en el uso más frecuente de adjetivos, favoreciendo también el uso de formas estándar. El diálogo, tal como se definió anteriormente, es altamente interactivo (una pregunta rápida, una respuesta rápida). Carece de la misma organización, proceso mental y ornamentación que caracterizan a la argumentación y a la descripción, y por lo tanto tiende a favorecer el uso de variantes no estándar. En términos de propósito, tanto la narrativa como el diálogo suelen ser de mayor contenido afectivo, mientras que la argumen- 
tación, la descripción y la exposición tienen como base el proveer información. Finegan y Biber sugieren que los registros informativos favorecen una mayor claridad en la expresión y por lo tanto tienden a favorecer un uso mayor de variantes estándar. El último parámetro que mencionan es el del nivel de referente que comparten los hablantes o lectores. En muchas de las narrativas los hablantes conocidos compartían muchos de los referentes conmigo, especialmente por ser hablantes que forman parte de mi familia y de mi círculo de amigos íntimos. Cuando se comparten los elementos referenciales en una conversación, se facilita el nivel de naturalidad entre los hablantes, favoreciendo así el uso de formas no estándar. Las narrativas también tienden a acaparar la atención del interlocutor y favorecen un lenguaje mucho más afectivo y emotivo.

Tanto el tipo de situación como el tipo de discurso son valores altamente significativos en el análisis estadístico. Ambos factores son diferentes pero parecen relacionarse en algunos aspectos. Por ejemplo, en el presente estudio el diálogo apareció únicamente en la conversación individual y en la conversación en grupo y no así en la presentación oral, mientras que la argumentación apareció solamente en la conversación individual. El tipo de situación se relaciona al concepto de diseño del lenguaje de acuerdo a la audiencia, del que habla Bell (1984), mientras que el tipo de discurso es independiente de la audiencia. Sin embargo, tanto el tipo de audiencia como el tipo de discurso sirven para explicar diferencias lingüísticas. Estas observaciones sirven de apoyo al estudio de Finegan y Biber (1994), en el que se muestran y se explican distintos registros para mostrar las diferencias en el uso del lenguaje, recordando que los registros, bien sean orales o escritos, no existen independientemente de la audiencia.

\subsubsection{Tema de conversación}

La variable tema de conversación mostró grandes complicaciones para el análisis del presente estudio, quizás porque el tema de conversación se relaciona directamente al tipo de discurso y viceversa. Resulta un tanto difícil controlar la manera en la que un hablante manejará un tema específico. En el caso de las conversaciones individuales se procuró que todos los hablantes participaran de los mismos temas (niñez, familia, amigos, momento de vergüenza, momento triste, pasatiempos, momento de peligro, trabajo, escuela, aborto, pena de muerte, política, momento feliz). Los temas sobre "niñez" y sobre "un momento de vergüenza" favorecieron el uso de variantes no estándar más que otros temas de conversación con probabilidades de ocurrencia de .73 y .74 para el estudio de la vibrante múltiple (ver tabla 5). Ambos temas resultaron ser ricos en narrativas, tipo de discurso que a su vez favoreció también el uso de variante no estándar más que otros géneros discursivos. El tema de "política," por otro lado, favoreció mucho menos el uso de variantes no estándar con una probabilidad de ocurrencia de .33 para el estudio de la vibrante múltiple. Para el tema de "política," los hablantes se referían a elementos controversiales sobre el ambiente político en Puerto Rico durante un año electoral. Este tema favoreció el estilo argumentativo y a su vez el uso de variantes estándar. Cabe mencionar que para el tema de política, los hablantes se veían comprometidos a hablar sobre sus inclinaciones políticas, lo cual en la mayoría de los casos se considera un asunto delicado aún entre familiares y amigos. Hubo ocasiones en que algunos hablantes esquivaron el tema de la política sin manifestar su opinión personal. Mucha gente que vive en Puerto Rico y que trabaja con el gobierno estatal tiende a ser muy cuidadosa al hablar sobre sus inclinaciones político-partidistas, temiendo perder sus empleos si se les descubre que favorecen al partido de oposición. De manera paradójica, a esta misma gente les encanta hablar sobre la política, especialmente durante un año de elecciones.

Los temas controversiales que favorecen la argumentación, como es el caso del tema político, tienden a producirse con mayor lentitud en la conversación, tal como se observó en la sección anterior. En este caso 
los hablantes necesitan pensar y escoger sus argumentos con mucho cuidado, ya que están poniendo en evidencia su capacidad para desarrollar un pensamiento crítico. También en este tipo de situaciones el hablante teme ser juzgado por su interlocutor. En general, todos los temas controversiales como la política, la pena de muerte y el aborto favorecieron el discurso argumentativo. En este sentido parece ser que tanto el tema de conversación, como el tipo de discurso que usa el hablante y la rapidez con que se produce un discurso son factores que se relacionan entre sí.

En oposición a los temas controversiales se pueden mencionar otros temas como el de "la niñez," el cual se basó en la mayoría de los casos en recuerdos de alto contenido afectivo, y el tema sobre "un momento feliz" caracterizado en su mayoría por experiencias de tipo risible. Estos temas favorecieron por su parte el género narrativo y a su vez el uso de variantes no estándar.

Los resultados para la variable tema de conversación apoyan las observaciones del estudio de Rickford y McNair-Knox (1994), en el cual sugieren que ciertos temas tienden a favorecer el uso de formas no estándar más que otros. Para una futura investigación sugiero que se combinen las variables tema de conversación y tipo de discurso, o que se cree una conversación mucho más dirigida o controlada, en la que se experimente con un mismo tema a partir de géneros discursivos diferentes. Por ejemplo si se escoge el tema sobre la escuela, que se le dé la oportunidad al hablante para que narre anécdotas sobre su vida escolar, para que describa sus clases, para que evalúe el sistema educativo, etc.

\section{Conclusión}

A través del presente estudio se ha mostrado la importancia que tiene el incorporar otras variables estilísticas, fuera de las tradicionales, como parte de la investigación sociolingüística. Los hablantes que participaron en las grabaciones, los cuales pertenecían a un mismo grupo social y compartían muchas características entre sí, mostra- ron variación lingüística de acuerdo a la situación en la que se encontraron, el tema de su conversación, el tipo de discurso que utilizaron en dicha conversación y la relación [+/- familiar] que tenían con su interlocutor. Junto a las variables estilísticas que se analizaron, sería necesario además para el análisis cualitativo el detenerse a observar la "historia" del hablante, su profesión, su posición de poder dentro de su ambiente de trabajo y sus relaciones interpersonales para tener un cuadro más completo y preciso que sirva para explicar su comportamiento lingüístico.

Las dos variables fonológicas que se incorporaron para el análisis resultaron ser ideales para examinar las cuatro variables estilísticas que se incluyeron para este estudio. La manera en la que se distribuyeron las variantes estándar y no estándar a lo largo del análisis estadístico muestran como se asocian dichas variantes con determinados tipos de situaciones, temas, relaciones personales y discursos.

\section{nOTAS}

${ }^{1}$ Los factores linguiísticos que se examinaron para el estudio de la (r) en posición final de sílaba fueron: el contexto fonológico, sílaba tónica vs. sílaba átona, el valor morfemático de (r) y el número de sílabas para cada una de las palabras. Para el anális estadístico se eliminaron los casos posibles de asimilación fonológica, cuando a la (r) final de sílaba le seguía una consonante líquida o vibrante (e.g., por la, llegar rápido). Cabe mencionar que López-Morales (1983) no eliminó en su estudio dichos contextos fonológicos. En el factor "valor morfemático de (r)" se contrastan los infinitivos en oposición a otros posibles contextos; porque se codificó bajo una categoría diferente, dada la frecuencia de esta palabra en el discurso y por el hecho particular que dicha palabra favorece la aspiración y elisión de (r) hasta cierto punto.

${ }^{2}$ Los factores linguiísticos que se examinaron para el estudio de la variante múltiple (rr) fueron: el contexto fonológico, la sílaba tónica vs. la átona y el tipo de palabra. El grupo de factores "tipo de palabra" incluye nombres propios.

"Se incluyó el grupo de factores "tipo de palabra" porque tenía la intuición de que los nombres propios se tienden a pronunciar con mucho más cuidado.

${ }^{4}$ Los factores sociales que se incorporaron para este estudio fueron: el origen de los padres (urbano, rural o mixto), el nivel educativo de los participantes, el nivel educativo más alto entre el padre o la madre con los que vive o vivía el hablante, la edad y el sexo. 


\section{- OBRAS CITADAS}

Bell, Allan. 1984. Language Style as Audience Design. Language and Society 13: 145-204.

Finegan, E., y D. Biber. 1994. "Register and Social Dialect Variation: An Integrated Approach.” Ed. Biber y Finegan. Sociolinguistic Perspective on Register, New York: Oxford UP. 315-47.

Hammond, Robert. 1987. Un estudio cuantitativo y teórico de la r velar en Puerto Rico. Káñina, $R e$ vista de Artes y Letras, Universidad de Costa Rica 11: 163-73.

López-Morales, Humberto. 1983. Estratificación social del español de San Juan de Puerto Rico. México: Universidad Autónoma de México.

Medina-Rivera, Antonio. 1996. "Discourse Genre, Type of Situation and Topic of Conversation in Relation to Phonological Variables in Puerto Rican
Spanish.” Sociolinguistic variation: Data, Theory, and Analysis. Ed. J. Arnold et al. Stanford, CA: CSLI Publications. 209-22.

Medina-Rivera, Antonio. 1997. Phonological and Stylistic Variables in Puerto Rican Spanish. Ph.D. Dissertation, U of Southern California.

Milroy, James y L. Milroy. 1985. "Linguistic Change, Social Network and Speaker Innovation.” Journal of Linguistics 21: 339-84.

Navarro-Tomás, Tomás. 1948. El español en Puerto Rico. Río Piedras: Universidad de Puerto Rico.

Poplack, Shana. 1979. Function and Process in a Variable Phonology. Ph.D. Diss. U of Pennsylvania.

Rickford, J., y F. McNair-Knox. 1994. "Addressee and Topic-Influenced Style Shift: A Quantitative Sociolinguistic Study.” Biber y Finegan, eds. 235-76.

Tabla 5 PROBABILIDADES ESTADISTICAS DE VARBRUL (step up and down)

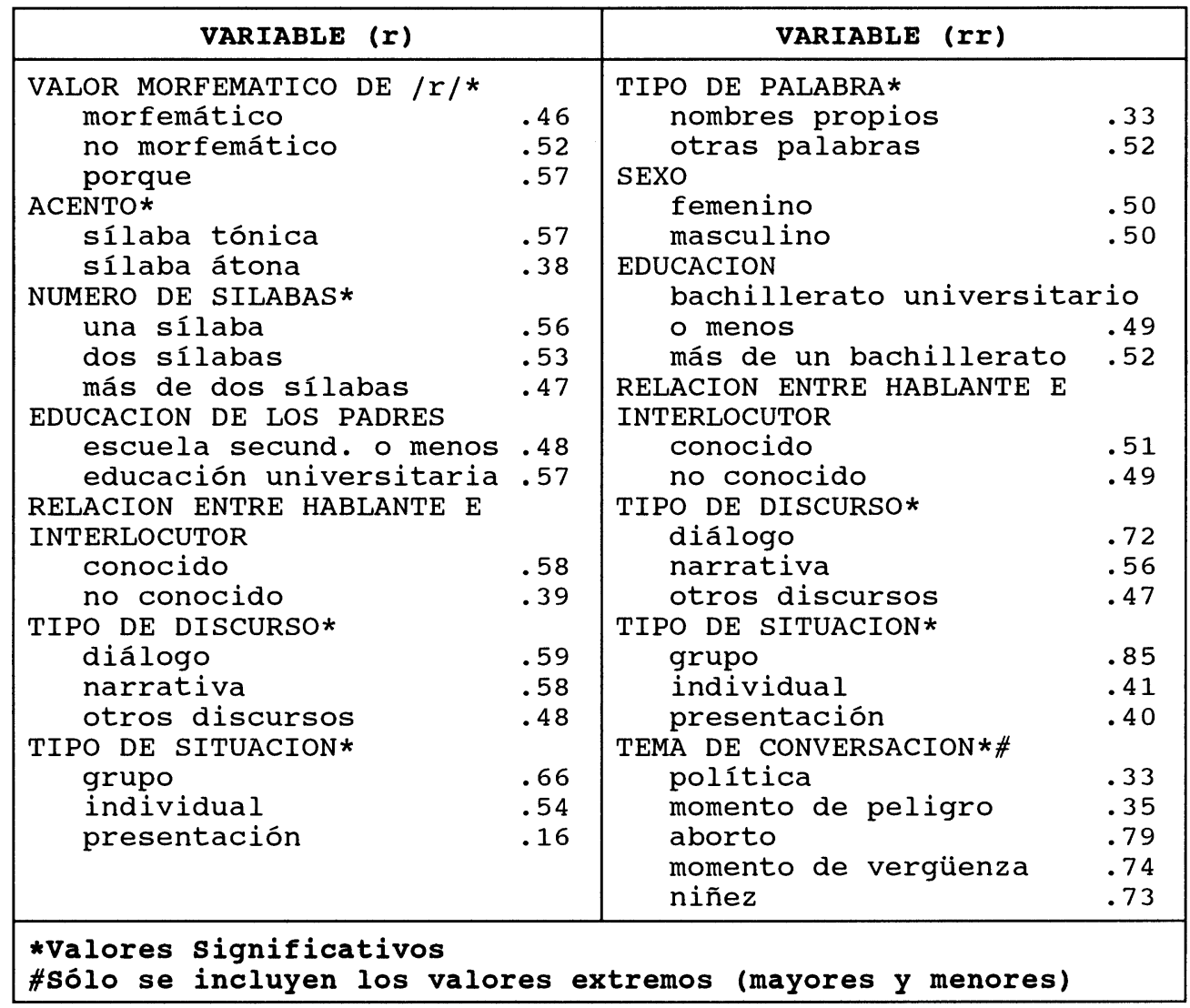

